\title{
Analysis of tissue PLA2R efficacy in evaluating the prognosis of idiopathic membranous nephropathy in the background of different serum anti-PLA2R levels
}

\author{
Yuemeng Sun ${ }^{1}$, Ping Lan ${ }^{1}$, Jie Feng ${ }^{1}$, Zhigang Wang ${ }^{1}$, Chao Liu ${ }^{1}$, Yan Li $^{1}$, Liyi Xie ${ }^{1}$, and \\ Xiaoyang $\mathrm{Yu}^{1}$ \\ ${ }^{1}$ Affiliation not available
}

December 30, 2020

\begin{abstract}
Objective To verify serum PLA2R antibody and glomerular PLA2R antigen expression in membranous nephropathy as well as to explore their relationship with clinical presentation and disease prognosis. Methods We retrospectively analyzed 155 patients clinical figures who were diagnosed with primary membranous nephropathy by kidney biopsy. Patients were divided into 6 groups according to their serum PLA2R antibody or glomerular PLA2R antigen positiveness and the level of serum PLA2R antibody titer. Both clinical features and pathological characteristics were recorded, and remission rate as well as time to response were compared among groups. Correlation between clinical figures and titer of PLA2R antibody or semi-quantity of PLA2R antigen were detected. Results Among patients with positive serum PLA2R antibody and tissue PLA2R antigen, higher baseline PLA2R antibody levels were associated with lower remission rate and longer remission time. A positive correlation between time to partial remission and serum PLA2R antibody titer was found. Among patients with serum PLA2R antibody titer $<150 \mathrm{U} / \mathrm{L}$, there were shorter remission time in negative tissue PLA2R antigen group compared with positive tissue PLA2R antigen, and a positive correlation between time to complete remission and semi-quantity of tissue PLA2R antigen was found. Conclusion Both glomerular PLA2R antigen and serum PLA2R antibody play a role in disease presentation and prognosis in primary membranous nephropathy. Glomerular PLA2R antigen has a major role on disease prognosis when serum PLA2R antibody titer is less than $150 \mathrm{U} / \mathrm{L}$, while serum PLA2R antibody has predominant role in MN prognosis when serum PLA2R antibody titer is above $150 \mathrm{U} / \mathrm{L}$.
\end{abstract}

\section{Hosted file}

$\backslash$ begin $\{\mathrm{CJK}\}\{\mathrm{UTF} 8\}$ \{gbsn\} \end\{CJK\}\selectlanguage\{english\}submission -primary membranous nephropathy and } available at https://authorea.com/users/386175/articles/501522-analysis-of-tissue-pla2refficacy-in-evaluating-the-prognosis-of-idiopathic-membranous-nephropathy-in-thebackground-of-different-serum-anti-pla2r-levels

\section{Hosted file}

Table submission -MN.pdf available at https://authorea.com/users/386175/articles/501522analysis-of-tissue-pla2r-efficacy-in-evaluating-the-prognosis-of-idiopathic-membranousnephropathy-in-the-background-of-different-serum-anti-pla2r-levels

\section{Hosted file}

figure submission-MN.pdf available at https://authorea.com/users/386175/articles/501522analysis-of-tissue-pla2r-efficacy-in-evaluating-the-prognosis-of-idiopathic-membranousnephropathy-in-the-background-of-different-serum-anti-pla2r-levels 